\title{
Analysis of the Driven Types of Consumer Silence Behaviors in Scenarios of Service Failure
}

\author{
Wang Wenfeng ${ }^{1}$ \\ ${ }^{1}$ School of International Business, Jinan University, Zhuhai, 519070
}

\begin{abstract}
It will have significant practical and theoretical value that when we pay attention to the consumer silence behaviors in scenarios of service failure. The paper begins with the exploration of the five types of silence behaviors and their causes, including defense-driven,

helplessness-driven, suspicion- driven, pro-social-driven and disregard-driven behaviors, followed by appropriate management strategies proposed on the basis of an analysis of the impact on those types of behaviors on brand loyalty.
\end{abstract}

Keywords: Service failure; consumer silence; brand loyalty

\section{INTRODUCTION}

As indicated by earlier psychology literatures, the delivery of bad messages is often accompanied such bad feelings that people are generally reluctant to do so, a phenomenon which is known as the mum effect(Rosen, 1970). Consumer silence behavior refers to the behavioral state in which consumers keep to themselves the problems in service instead of complaining to the service businesses or voicing their complaints on the spot. It will have significant practical and theoretical value that when we pay attention to the consumer silence behaviors in scenarios of service failure.

From a practical perspective, it will be good for service recovery in the first place. It is generally believed that, in order to improve service quality and customer loyalty, it is necessary for service businesses to take appropriate recovering measures for service failures. In most circumstances, however, consumers do not give the opportunities for service businesses to approach them and recover services (Gelbrich, 2010), which calls for attentions to be paid to such silent consumers before service failure can be promptly noticed and recovered. Secondly, it is good for customer retention. As shown by a survey report released by TARP (Technical Assistance Research Programs, Inc.) that in the event of service failure, as many as 95 percent of customers will not make a complaint to service businesses and approximately 50 to $90 \%$ of such silent people are likely to switch to a business competitor. Of course it is obviously extremely unwise to ignore this silent group when it becomes increasingly difficult to garner new customers today. Finally, it is good for control of negative effects. Despite how silent consumers hold direct complaints back from service businesses, negative word of mouth may be spread or complaints made to a third party, having a serious impact on service businesses (Davidow and Dacin, 1997), . Especially with the development of mobile Internet, online negative information just gets more and more destructive.

From a theoretical perspective, the consumer silence behaviors in scenarios of service failure first will be good for the underdeveloped study of customer complaint behaviors. Current studies of consumer silence behaviors focus on customer complaints that ensue from service failures, for example, Davidow and Dacin (1997) divide consumer behaviors in scenarios of service failure into four types: voice; negative word of mouth, boycott, and third-party complaints. However, in studies of customer complaints, more attention is paid to voice than to silence behaviors, the latter of which is only handled as a special type of complaints. Therefore, attention paid to consumer silence behaviors will help expand our vision in studying customer complaints. Secondly, it will help enrich the study of silence behaviors. Currently, studies of silence behaviors have drawn much attention and have earned recognition in the field of organizational behavior (Zheng Xiaotao, 2008). As interactions between service subjects play an increasing role, customers can be seen as "prospective employees" to companies. The study of consumer silence behaviors will help enrich and deepen the theories about silence behavior.

\section{The driven types of consumer silence behaviors in scenarios of service failure and their causes}

It is found by conducting an open questionnaire survey of Chinese consumers, pre-test, exploratory factor analysis and confirmatory factor analysis based on existing research results that in scenarios of service failure, there are five driven types of consumer silence behaviors as follows:

\subsection{Defense-driven silence behavior}

It refers to the defensive silence behavior that consumers have under social pressure or for the avoidance of dispute and revenge in scenarios of service failure. The items included by it are "Worrying about giving a picky impression and losing face", "Complaining is ungracious", "Do not want to be the black sheep while other customers are silent" "I am worried about disputes arising from complaining". The defensive silence behavior, as a means to prevent psychological stress imposed by cultural and social environment, is rooted in profound cultural values. For example, Chiu (1988) believes that Chinese people 
attach so much importance to "face" that in order to avoid conflicts, they opt not to complain. In addition, it takes firm root among the masses that he who stands out usually bears the brunt of attack, hence herd behavior is the safest option in the eyes of most consumers. It is clear that such silence is kept to meet the need for psychological security.

\subsection{Helplessness-driven silence behavior}

It refers to a helpless choice for silence made by consumers in scenarios of service failure because there was no complaint channel or good complaint channel. The items included by it are "Do not know whom the complaint can be filed to", "Do not know where the complaint can be filed", "The service business does not have a dedicated complaint department," "It is too troublesome to go through the complaint procedures". The reasons for helplessness-driven silence behavior can be objective and subjective. Objectively, consumers, when encountering service failures, have no idea about how to express what they think due to the lack of communication between service businesses and them. They would even helplessly see the buck being passed among workers concerned. Subjectively, consumers would quit when they figure out the high cost of complaint due to the formalities. (Richins, 1982). Some consumers see service failure as a doom and feel reluctant to complain (Yau and Oliver, 1988). Such silence behavior is a kind of helpless choice made by consumers disappointed about process fairness.

\subsection{Suspicion-driven silence behavior}

It refers to a behavioral expression given by consumers, in scenarios of service failure, to backdown out of distrust of service businesses or personnel. The items included by it are "Even if I file the complaint, the service would not be improved" "Even if I tell it what I think, the service business will not listen" "Complaints would not get handled in a timely manner", "Complaints would just go down the drain". Such silence behaviors may be motivated by the lack of consumer trust in service businesses, resulting in its poor confidence in complaint earnings and success. Consumers are silence prone when they do not believe that they would get what they deserve after complaining, or doubt that the service organization would listen to customers and solve problems.

\subsection{Pro-social-driven silence behavior}

It refers to a behavior that consumers, instead of voicing direct complaints to service organization or employees, are always understanding and tolerant in scenarios of service failure. The items included by it are "Service failure are excusable" "I would put myself in other's shoes and remain considerate of the service business" "They are kind and a little mistake does not matter," "I do not want to give them troubles". Such silence behavior is motivated by altruism and cooperation ( Yao Shengjuan et al., 2009). In the process to attribute service failures, consumers often convince themselves that service businesses have nothing to do with service failures, thus consumers would go for tolerance and understanding in silence (Chebat et al,2005). Of course, empathy has a greater impact on consumers in silence of such type. (Harris and Mowen, 2001)

\subsection{Disregard-driven silence behavior}

It refers to silence behavior that consumers have disregardful of the harm and loss caused by service failures. The items included by it are "It is too trifling to make a big deal of it" "The service failure is not substantially harmful to me", "Though not perfect, the quality of service is acceptable", and "I am not a customer regular enough to look for trouble". Richins (1983) suggests that the complaint tendency of consumers is affected by complaint interests perceived by them. The less serious they think the problem is, the less energy they would spend on it, the more likely they will keep silence. In addition, consumers may opt for disregard-driven silence when they do not consider the service brand important.

\section{Impact of consumer silence behaviors in scenarios of service failure on brand loyalty}

Service failure means that services delivered fail to meet customer expectations (Holloway and Beatty, 2003). Thus, service failure will inevitably result in cognitive dissonance to some extent. According to the cognitive dissonance theory, when cognitive dissonance occurs to someone, it would have a pressure on dissonance improvement and on avoidance of dissonance deterioration, leading to cognitive or behavioral changes (Richins, 1983).

However, silence behaviors of different types, as driven by different factors, will put consumers in cognitive dissonance to different extent, and have different impact on service brand loyalty. Defense-driven silence mainly stems from the consumer need for psychological security and it is a means of self protection for themselves. Therefore, the impact on consumers and service brand loyalty primarily comes from service failure itself and is relatively small. Although it may trigger unpleasant feelings due to service failures and cognitive dissonance, pro-social-driven silence would impel consumers to be understanding and tolerant to mitigate the pressure caused by such cognitive dissonance, and therefore have a small impact on the original brand loyalty. As for disregard driven silence, it will not undermine the brand loyalty if the original brand relationship itself is temporary; and if the losses from service failure are insignificant to consumers, there will be no significant cognitive dissonance and no serious impact on the original brand loyalty. Different from those above, helplessness- and suspicion-driven silence behaviors are the result of no channels or no good channels for consumers to file complaints or the result of distrust of service brands and they are closely associated with service brands. Therefore, in scenarios of service failure, those two types may amplify consumers' negative experience of service failure, leading 
to growing cognitive dissonance, and thus undermining brand loyalty.

\section{Management Revelations and Policies}

As shown by the foregoing analysis, service businesses must not ignore consumer silence behaviors. It is necessary for managers to identify the motivations behind consumer silence behaviors and tell the types of those behaviors before they can take timely, effective and comprehensive measures for service recovery, and further improve their service system to avoid losing customers. (1) For defense-driven silence behavior, service businesses should dismiss the misgivings as much as possible that consumers have if they plan to complain. For example, they may design a Wechat(SMS) platform for consumers to file complaints and define responsibilities for failures to be borne by consumers and service businesses. (2) For helplessness-driven silence behavior, service businesses should focus on "process fairness" for consumers, for example, specifying the departments and personnel handing consumer complaints; giving more publicity so that consumers know where they can file complaints; simplifying complaint procedures and improving consumers' sense of control and so on. (3) For suspicion-driven silence behavior, the key is that service businesses should seek to enhance consumer confidence. For example: making clear service commitment and informing consumers of them; establishing a good reputation and brand image when dealing with complaints; developing measures to encourage consumer complaints.

(4) For pro-social-driven silence behavior, service businesses should prevent service failures as practical as possible. For example, it may strengthen the management for critical service-failure-prone period; it may develop a "zero defects" corporate culture. (5) For disregard-driven silence behavior, service businesses should focus on customer relationship management. For example, it may classify customers and strengthen its ties with VIP customers.

\section{CONCLUSIONS}

Under the background of Chinese culture, the following research conclusions could be drawn: There are five types of consumer silence behaviors in scenarios of service failure; different Silence behaviors driven by different factors had different impact on service brand loyalty. In the future, researchers should pay attention to the different types of silence behavior under different cultural background and the causes behind.

\section{Acknowledgements}

This study was supported by a grant from the Foundation of Ministry of Education of the people's Republic of China (No.12YJC630212)

\section{References}

[1] Rosen S, Tesser A. "On Reluctance to Communicate Undesirable Information: The MUM Effect," Sociometry, 33, pp.253-263,1970.

[2] Gelbrich K. "Anger, Frustration, and Helplessness after Service Failure: Coping Strategies and Effective Informational Support," Academy of Marketing Science, 38 (5), pp 567-585,2010.

[3] Davidow M, Dacin P A. "Understanding and Influencing Consumer Complaint Behavior: Improving Organizational Complaint Management," Advances in Consumer Research, 24(1), pp 450-456, 1997.

[4] Zheng Xiaotao, Ke Jianglin, Shi Jintao, Zheng Xingshan. "Measurement of the Silence of the Employees in Chinese Background and the Impact of Trust on It," Psychological Science. 40(2), pp 219-227,2008.

[5] Chiu C Y, Tsang S C, Yang C F. "The Role of Face Situation and Attitudinal Antecedents in Chinese Consumer Complaint Behavior," Journal of Social Psychology, 128(2), pp 173-180,1988.

[6] Richins M L. "An Investigation of Consumers" Attitudes toward Complaining," Advances in Consumer Research, 9(1), pp 502-506,1982.

[7] Yau, Oliver H M. "Chinese Cultural Values: Their Dimensions and Marketing Implications," European Journal of Marketing, 22(5), pp 44-57,1988.

[8] Yao Shengjuan, Deng Yanan, Zheng Junhu. "The Cultural Root of The Silence Behaviors of the Employees in Chinese Background," East China Economic Management, 23(6): pp135-138, 2009.

[9] Chebat J C, Davidow M, Codjovi I. "Silent Voices: Why Some Dissatisfied Consumers Fail to Complain," Journal of Service Research, 7(4), pp 328-342, 2005 .

[10] Harris E G, Mowen J C. "The Influence of Cardinal-,Central-,and Surface-level Personality Traits on Consumers' Bargaining and Complaint Intentions," Psychology\& Marketing, Hoboken, 18(11), pp 1155-1185, 2001.

[11] Richins M L . "An Analysis of Consumers Interaction Styles in the Marketplace," Journal of Consumer Research, 10 (1), pp 73-82,1983.

[12] Holloway BB, Beatty SE. "Service Failure in Online Retailing: A Recovery Opportunity," Journal of Service Research, 6(1), pp 92-105, 2003. 